Article

\title{
Magnetic Field in Nuclear Collisions at Ultra High Energies
}

\author{
Vitalii A. Okorokov \\ National Research Nuclear University MEPhI, 115409 Moscow, Russia; VAOkorokov@mephi.ru
}

Received: 31 May 2019; Accepted: 28 June 2019; Published: 2 July 2019

check for updates

\begin{abstract}
The magnetic field created in proton-proton and nucleus-nucleus collisions at ultra-high energies are studied with models of point-like charges and hard sphere for distribution of the constituents for vacuum conditions. The various beam ions are considered from light to heavy nuclei at energies corresponding to the nominal energies of the proton beam within the projects of further accelerator facilities high-energy Large Hadron Collider (HE-LHC) and Future Circular Collider (FCC). The magnetic-field strength immediately after collisions reaches the value tens of $\mathrm{GeV}^{2}$, while in the approach with point-like charges, some overestimate the amplitude of the field in comparison with more realistic hard-sphere model. The absolute value of the magnetic field rapidly decreases with time and increases with growth of atomic number. The amplitude for $e B$ is estimated at level $100 \mathrm{GeV}^{2}$ to provide magnitude for quark-quark collisions at energies corresponding to the nominal energies of proton beams. These estimations are close to the range for onset of $W$ boson condensation.
\end{abstract}

Keywords: high-energy nuclear collisions; quark-gluon matter

PACS: 25.75.-q; $25.75 . \mathrm{Nq}$

\section{Introduction}

According to the Biot-Savart law, the current, i.e., moving charge, Creates a magnetic field $(\vec{B})$. Therefore, the collisions of charged particles in human-made accelerator facilities or in cosmic rays generate the magnetic field whose strength can achieve a very large value. This field appears just after collision and, consequently, can influence all stages of space-time evolution in the final-state system. The influence of $\vec{B}$ and the corresponding electric filed $(\vec{E})$ can be essential for the phase diagram of the matter created in the final state and for transition processes at sufficiently large strength of this external Abelian (electro)magnetic field. Also, it can lead to some new features for dynamics of multiparticle production. In general, the maximum of the absolute value $B \equiv|\vec{B}|$ will increase with growth of the energy of the incoming particles and, consequently, the amplification of the influence of (electro)magnetic field on the various properties of the final state in the domain of very high energies can be expected. As a consequence, the study of the possible influence of external Abelian (electro)magnetic field created through the collisions of relativistic particles on the interaction process is important for both the strong interaction and the electroweak sector. Collisions of particles with ultra-relativistic energies provide a unique possibility to study the wide set of physical effects related to the very strong electromagnetic fields at controlled conditions. On the other hand, such investigations can shed new light on the physical mechanisms that may have produced magnetic fields in the early universe. Therefore, studies of extremely strong electromagnetic field in particle collisions with ultra-high energies can be important for the physics of fundamental interactions, cosmology, and relativistic astrophysics, i.e., they have an interdisciplinary value. 


\section{Definitions and Notations}

\subsection{Models for Magnetic Field}

In the simplest approach, one can assume, as in [1], that the colliding objects are point-like particles with charges Ze, where $e \equiv|e|$ is the magnitude of electron charge. Let the objects move along the $Z$ axis at impact parameter $\vec{b}$. Then, time evolution of $B$ at the center of the collision can be described by the following equation [2]

$$
B(t)=B_{0}\left[1+\left(t / t_{0}\right)^{3 / 2}\right]^{-1}, \quad e B_{0}=8 \mathrm{Z} \alpha_{\mathrm{EM}} \sinh \left(y_{0}\right) / b^{2}, \quad t_{0}=b /\left[2 \sinh \left(y_{0}\right)\right] .
$$

Here $\alpha_{\mathrm{EM}}$ is the electromagnetic constant and $y_{0}$ is the rapidity of the incoming particles in the center-of-mass system. In relativistic energy domain $\left(p_{0} \gg m\right)$ the relations $\sinh \left(y_{0}\right)=\gamma_{0} \beta_{0, z} \approx$ $\sqrt{s} / 2 m$ result in

$$
e B_{0} \propto \sqrt{s},
$$

where $p_{0}, \gamma_{0}, \beta_{0, z}$ and $m$ is the momentum, Lorentz factor, velocity along the $Z$ axis, and mass of incoming particle in the frame considered; $s$ is standard Mandelstam invariant variable.

The magnetic field at a point $\vec{x}=\left(\vec{x}_{\perp}, z \vec{e}_{Z}\right)$ created by an object (proton/nucleus) with finite size and a charge $Z e$, moving in the positive $(z>0)$ direction of the $Z$ axis from the starting point of the transverse plane $\vec{x}_{\perp}^{\prime}=\left.\vec{x}_{\perp}\right|_{t=0}$, can be obtained either with help of appropriate conversation of electric field of a given charged object, or based on the Liénard-Wihert potentials [3]. In this work the magnetic field created immediately after the collision is studied, i.e., $\vec{B}$ at times $t>0$, where $t=0$ is the collision moment. Therefore $\vec{B}$ can be written as follows [3]:

$$
\vec{B}=\sum_{i=+,-} \sum_{j=\mathrm{s}, \mathrm{p}} \vec{B}_{j}^{i}
$$

where $\vec{B}_{\mathrm{s}}^{ \pm}, \vec{B}_{\mathrm{p}}^{ \pm}$are the contributions of magnetic fields from the spectator constituents $\left(\mathrm{N}_{\mathrm{s}}\right)$ and participating constituents $\left(\mathrm{N}_{\mathrm{p}}\right)$, moving in the positive (negative) direction of the $Z$ axis. The contributions from spectators and participants can be estimated with help of the following equations [3]

$$
\begin{gathered}
e \vec{B}_{\mathrm{s}}^{ \pm}\left(\tau, \tilde{y}, \vec{x}_{\perp}\right)= \pm \mathrm{Z} \alpha_{\mathrm{EM}} \sinh \left(y_{0} \mp \tilde{y}\right) \int d^{2} \vec{x}_{\perp}^{\prime} \rho_{ \pm}\left(\vec{x}_{\perp}^{\prime}\right)\left[1-\theta_{\mp}\left(\vec{x}_{\perp}^{\prime}\right)\right] \vec{\zeta}^{ \pm}\left(\tau, \tilde{y}, \vec{x}_{\perp}, \vec{x}_{\perp}^{\prime}, y_{0}\right), \\
e \vec{B}_{\mathrm{p}}^{ \pm}\left(\tau, \tilde{y}, \vec{x}_{\perp}\right)= \pm \mathrm{Z} \alpha_{\mathrm{EM}} \int d^{2} \vec{x}_{\perp}^{\prime} \rho_{ \pm}\left(\vec{x}_{\perp}^{\prime}\right) \theta_{\mp}\left(\vec{x}_{\perp}^{\prime}\right) \int_{-y_{0}}^{y_{0}} d y_{\mathrm{p}} f\left(y_{\mathrm{p}}\right) \sinh \left(y_{\mathrm{p}} \mp \tilde{y}\right) \vec{\zeta}^{ \pm}\left(\tau, \tilde{y}, \vec{x}_{\perp}, \vec{x}_{\perp}^{\prime}, y_{\mathrm{p}}\right), \\
\vec{\zeta}^{ \pm}\left(\tau, \tilde{y}, \vec{x}_{\perp}, \vec{x}_{\perp}^{\prime}, \kappa\right)=\frac{\left(\vec{x}_{\perp}^{\prime}-\vec{x}_{\perp}\right) \times \vec{e}_{Z}}{\left[\left(\vec{x}_{\perp}^{\prime}-\vec{x}_{\perp}\right)^{2}+\tau^{2} \sinh (\kappa \mp \tilde{y})^{2}\right]^{3 / 2} .}
\end{gathered}
$$

Here $y_{p}$ is the rapidity of participants $N_{p}$ in the laboratory reference frame, which coincides with the center-of-mass system for collider beams, $\tau=\sqrt{t^{2}-z^{2}}$ and $\tilde{y}=0.5 \ln [(t+z) /(t-z)]$ is the time (proper time) and rapidity of a constituent in the object rest system, $\rho_{ \pm}\left(\vec{x}_{\perp}^{\prime}\right)$ is the constituent density. It is considered that spectators $N_{s}$ do not (re)scatter and continue to move along the $Z$ axis with $y_{0}$ after the interaction. Within the hypothesis about negligible contribution of the newly produced particles to the $\vec{B}$ [3] the function $f(x)=[a \exp (a x)] /\left[2 \sinh \left(a y_{0}\right)\right]$ is entered to account for contributions only from $\mathrm{N}_{\mathrm{p}}$ presented in the initial state, where $a \simeq 0.5$ based on available experimental data [4].

In relativistic energy domain, the Lorentz factor $\gamma_{N} \simeq \sqrt{s_{N N}} / 2 m_{N} \gg 1$ and colliding objects (proton / nucleus) are strongly contracted in the longitudinal (Z) direction of their original size, where $m_{N}$ is the nucleon mass [5]. Therefore in the simple approximation "hard sphere" the constituent density is defined as $\rho_{ \pm}\left(\vec{x}_{\perp}^{\prime}\right)=3\left[R^{2}-\left(\vec{x}_{\perp}^{\prime} \pm \vec{b} / 2\right)^{2}\right]^{1 / 2} \theta_{ \pm}\left(\vec{x}_{\perp}^{\prime}\right) /\left(2 \pi R^{3}\right)$ for the charged object moving in a positive (negative) direction along the $Z$ axis, where normalization is the following 
$\left.\int d^{2} \vec{x}_{\perp}^{\prime}\right) \rho_{ \pm}\left(\vec{x}_{\perp}^{\prime}\right)=1$ and $\theta_{ \pm}\left(\vec{x}_{\perp}^{\prime}\right)=\theta\left[R^{2}-\left(\vec{x}_{\perp}^{\prime} \pm \vec{b} / 2\right)^{2}\right]$ are the projections of the colliding objects on the transverse plane with respect to the beam axis, $\theta(x)$ is the step function used for splitting $\mathrm{N}_{\mathrm{s}}$ and $\mathrm{N}_{\mathrm{p}}$ in the approach considered, $R$ is the radius of the beam object (proton/nucleus). Figure 1 shows in detail the collision geometry and parameters used for calculation of $\vec{B}$ with help of (2).

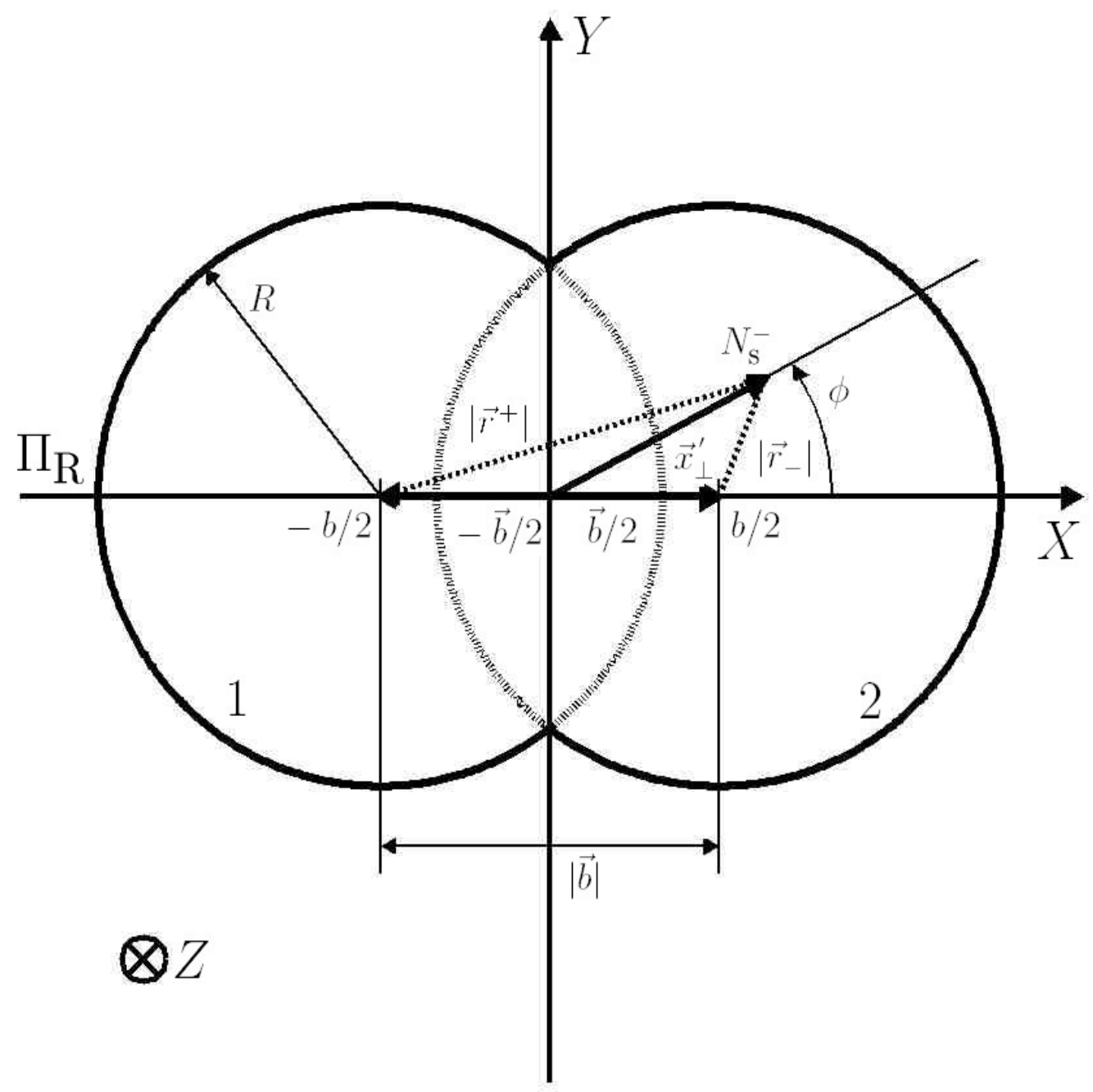

Figure 1. Detailed picture for collision geometry of two equal objects with finite size (proton/nucleus) in the plane transverse with respect to the beam axis $(Z)$ within hard-sphere model. The objects with radii $R$, moving in opposite directions (the object 1 -in the positive direction of $Z$, the object 2 -in negative), collide with the impact parameter $|\vec{b}|$. In this case, without loss of generality, the coordinate system is chosen in such a way that $X Z$ plane coincides with the reaction plane $\Pi_{R}$ and, consequently, the angle $\phi$ is the angle relative to $\Pi_{R}$. The region of the overlap of the two objects, shown by the dotted curve, contains participating constituents; the spectator constituents are outside the specified area. In the $X Y$ plane, the position of the spectator $N_{\mathrm{S}}^{-}$is characterized by the vector $\vec{x}_{\perp}^{\prime}$ relative to the origin of the coordinate system and by the vectors $\vec{r}_{-}^{+}=\vec{x}_{\perp}^{\prime} \pm \vec{b} / 2$ shown as the dashed lines with respect to the centers of the objects 1 and 2, respectively.

Within the hard-sphere approach and in the center of the secondary particle source, i.e., in the center of the overlap region $\left(\left|\vec{x}_{\perp}\right|=0\right.$ and $\left.\tilde{y}=0\right)$, the magnetic field points along the $Y$ axis: $\vec{B}=B \vec{e}_{Y}[3,6]$. This statement agrees well with the averaged results of event-by-event numerical 
calculations for various nucleus-nucleus collisions. The improvement of estimation of the $\vec{B}_{\mathrm{p}}^{ \pm}$provides the following analytic approximation [7]:

$$
e B \simeq 8 \mathrm{Z} \alpha_{\mathrm{EM}} \frac{b}{\tau^{3 / 2}} \exp \left(-y_{0} / 2\right)\left[\frac{\tilde{c} f(x)}{x R^{3 / 2}}+\frac{\exp \left(-3 y_{0} / 2\right)}{\tau^{3 / 2}}\right],
$$

which is valid for proper time range $\tau \in\left[\tau_{1}, \tau_{2}\right]$ with $\tau_{1}=R / \sinh \left(y_{0}\right)$ and $\tau_{2}=R$. In (4) the first term corresponds to the contribution of $\mathrm{N}_{\mathrm{p}}$ and the second one- to the contribution of spectators $\mathrm{N}_{\mathrm{s}}, \tilde{c} \simeq 0.075, x \equiv b / R$, the function $f(x)=\sum_{ \pm} \mp \sqrt{R} \int d^{2} \vec{x}_{\perp}^{\prime} \rho_{ \pm}\left(\vec{x}_{\perp}^{\prime}\right) \theta_{\mp}\left(\vec{x}_{\perp}^{\prime}\right) x^{\prime}\left|\vec{x}_{\perp}^{\prime}\right|^{-3 / 2}$ is calculated numerically [3]. At high energies $y_{0} \gg 1$ and the following analytic expressions can be derived for limit values of the magnetic field within the approximation (4):

$$
\begin{aligned}
\left.(e B)\right|_{\tau_{2}} & \approx 8 \mathrm{Z} \alpha_{\mathrm{EM}} \frac{\tilde{c} f(x)}{R^{2}} \exp \left(-y_{0} / 2\right) \\
\left.(e B)\right|_{\tau_{1}} & \left.\approx(e B)\right|_{\tau_{2}} \times \sinh ^{3 / 2} y_{0}\left[1+\frac{0.5 x}{\tilde{c} f(x)}\right]
\end{aligned}
$$

It should be noted that the analytic Equations (1) and (4) deduced within point-charge and hard-sphere approaches do not take into account any possible modifications due to matter produced in the final state, i.e., the models used here correspond to the $\vec{B}$ in vacuum.

\subsection{Beam Characteristics}

In the present paper, the energies are considered for the following international projects: the novel research infrastructure based on the Large Hadron Collider (LHC), which extends the current energy frontier by almost a factor of 2 is called the high-energy Large Hadron Collider (HE-LHC) project [8] and the integrated accelerator facility in a global context is called the Future Circular Collider (FCC) project which contains the work mode (FCC-hh) with proton and nuclei beams [9]. Both projects are essential parts of the next update of the European strategy for particle physics. The nominal energy for proton-proton collision within the HE-LHC project is $\sqrt{s_{p p}}=27 \mathrm{TeV}[8]$ and $\sqrt{s_{p p}}=100 \mathrm{TeV}$ for the FCC project [9]. Within colliding nuclei $\left(A_{1}, Z_{1}\right)+\left(A_{2}, Z_{2}\right)$ with nucleon numbers $A_{1}, A_{2}$ and charges $Z_{1} e, Z_{2} e$ in rings with magnetic field set for protons of momentum $p_{0, p}$ and mass $m_{p}$ [5], the colliding nucleon pairs will have an average beam energy and center-of-mass energy [10]

$$
\forall i=1,2:\left.E_{0, i}\right|_{m_{N} \approx m_{p}, m_{p} \ll p_{0, p}} \approx \mathrm{Z}_{i} E_{0, p} / \mathrm{A}_{i},\left.\quad \sqrt{s_{N N}}\right|_{m_{N} \approx m_{p}, m_{p} \ll p_{0, p}} \approx \sqrt{s_{p p} \times\left(\mathrm{Z}_{1} \mathrm{Z}_{2} / \mathrm{A}_{1} \mathrm{~A}_{2}\right)} .
$$

This work is devoted to the study of symmetric collisions. Some nuclei, from light to heavy, are considered to be beam particles for high-luminosity LHC [10]. It seems reasonable for complete information to consider the same nuclei as incoming particles at energies of the HE-LHC and FCC projects. Table 1 shows the essential kinematic parameters for various nuclei, where the first line corresponds to the $\sqrt{s_{p p}}=27 \mathrm{TeV}$ and second line to the $\sqrt{s_{p p}}=100 \mathrm{TeV}$ for each parameter considered.

Table 1. Kinematic parameters for various beams.

\begin{tabular}{cccccccc}
\hline \multirow{2}{*}{ Parameter } & \multicolumn{7}{c}{ Incoming Particle } \\
\cline { 2 - 8 } & ${ }^{\mathbf{1}} \boldsymbol{p}^{\mathbf{1 +}}$ & ${ }^{\mathbf{1 6}} \mathbf{O}^{\mathbf{8 +}}$ & ${ }^{\mathbf{4 0}} \mathbf{A r}{ }^{\mathbf{1 8}+}$ & ${ }^{\mathbf{4 0}} \mathbf{C a}^{\mathbf{2 0}+}$ & ${ }^{\mathbf{7 8}} \mathbf{K r}^{\mathbf{3 6 +}}$ & ${ }^{\mathbf{1 2 9}} \mathbf{X e}^{\mathbf{5 4 +}}$ & ${ }^{{ }^{\mathbf{2 0 8}} \mathbf{P b}^{\mathbf{8 2 +}}}$ \\
\hline$E_{0}, \mathrm{TeV}$ & 13.50 & 6.750 & 6.075 & 6.750 & 6.231 & 5.651 & 5.322 \\
& 50.00 & 25.00 & 22.50 & 25.00 & 23.08 & 20.93 & 19.71 \\
\hline$y_{0}$ & 10.31 & 9.576 & 9.472 & 9.576 & 9.492 & 9.396 & 9.341 \\
& 11.51 & 10.82 & 10.77 & 10.82 & 10.58 & 10.73 & 10.70 \\
\hline
\end{tabular}


The radius for beam particle is estimated as the radius of spherically symmetric object $\forall A: R=$ $r_{0} A^{1 / 3}$ with $r_{0}=(1.25 \pm 0.05)$ fm [11,12]. For $p+p$ interactions the quark-quark collisions $(q+q)$ can be also considered with the following estimations $\sqrt{s_{q q}} \sim \sqrt{s_{p p}} / 3$ [13-16] and $r_{q} \sim R_{p} / 3$ for constituent quarks.

The approach of the point-like charge can be applied for the finite-size object with characteristic linear scale (radius) $R$ at sufficiently large impact parameters $b \gg R$. The amplitude value of magnetic field from (1) can be re-written $e B_{0} \propto Z / x^{2} R^{2}$. Thus, one can see from (1) and (5) the amplitude $e B_{0}$ and extremes $\left.(e B)\right|_{\tau_{1}},\left.(e B)\right|_{\tau_{2}}$ of the hard-sphere model show similar behavior $X \propto Z / R^{2}, X \equiv e B_{0},\left.(e B)\right|_{\tau_{1}}$ or $\left.(e B)\right|_{\tau_{2}}$ with changes of the charge number and radius of beam particle and at fixed $x$. The following approximate empirical relation is valid for an isobar of a selected nucleus that is stable relative to $\beta$-decay [12]: $Z \approx A /\left(1.980+0.015 A^{2 / 3}\right)$. Then, one can derive the $Z$ - and $A$-dependence of the quantity $X$ within point-like particle and hard-sphere approaches for a magnetic field

$$
\begin{aligned}
& X(Z) \propto Z, \\
& X(A) \propto A^{1 / 3} /\left(1.980+0.015 A^{2 / 3}\right) .
\end{aligned}
$$

\section{Results}

Numerous phenomenological studies are devoted to the (electro)magnetic fields arising from nucleus-nucleus collisions (See, for instance, some review papers $[17,18]$ and references therein. The overview of electromagnetic probe production in heavy-ion collisions at relativistic energies can be found elsewhere [19].). However, up to now the papers are for the energies $\sqrt{s_{N N}}<7 \mathrm{TeV}$ [20] and they are usually focused on the heavy-ion collisions. In this work, the strength of external magnetic field is estimated within approaches of point-like charges (1) and hard sphere for collisions of the particles from Table 1 with $\sqrt{s_{N N}}$ corresponding to the nominal proton-proton collision energies within HE-LHC and FCC-hh projects for the first time.

The $B(t)$ dependence at the center of collision obtained within the approach of point-like charges shows rapid decrease with $t$ for any beam types from Table 1 , especially for $p+p$. The (1) allows the estimations for amplitude of the magnetic field and characteristic time depends on the beam type and $\sqrt{s_{N N}}$. The results for amplitude follow $e B_{0} \sim 20 \mathrm{GeV}^{2}$ for $p+p$ and this parameter is in the range $\sim(13-19) \mathrm{GeV}^{2}$ for rest nuclei; $t_{0} \sim 0.4 \times 10^{-4} \mathrm{fm} / c$ for $p+p$ and $(2-7) \times 10^{-4} \mathrm{fm} / c$ for other nuclei at $\sqrt{s_{p p}}=27 \mathrm{TeV}$. The corresponding estimations are $e B_{0} \sim 78 \mathrm{GeV}^{2}$ for $p+p$ and $\sim(49-71)$ $\mathrm{GeV}^{2}$ for rest of Table $1 ; t_{0} \sim 0.1 \times 10^{-4} \mathrm{fm} / c$ for $p+p$ and $(0.6-1.8) \times 10^{-4} \mathrm{fm} / c$ for other nuclei at $\sqrt{s_{p p}}=100 \mathrm{TeV}$. Values of the parameters $e B_{0}$ and $t_{0}$ are mostly growth for transition from $\mathrm{O}+\mathrm{O}$ to $\mathrm{Pb}+\mathrm{Pb}$ collisions. The quantitative results above correspond to the semi-central collisions $(x=1)$.

Figures 2 and 3 show the dependence $e B(\tau)$ for $p+p(\mathrm{a}), \mathrm{O}+\mathrm{O}(\mathrm{b}), \mathrm{Xe}+\mathrm{Xe}(\mathrm{c})$ and $\mathrm{Pb}+\mathrm{Pb}(\mathrm{d})$ collisions in central (dashed lines), semi-central (solid curves) and peripheral (dotted lines) events at $\sqrt{s_{p p}}=27$ and $100 \mathrm{TeV}$, respectively. These smooth curves are obtained within the hard-sphere model for the range $R / \sinh \left(y_{0}\right) \lesssim \tau \lesssim R$ with help of the analytic Equation (4). The magnetic field strength for particle species from Table 1 at HE-LHC (Figure 2) and FCC-hh (Figure 3) energies decreases fast with $\tau$ increase in a vacuum, especially for peripheral collisions. Such behavior of the $e B(\tau)$ dependence agrees with previous results at lower energies [6,7]. On the left boundaries of the temporary ranges, studies of the absolute value of the magnetic field reach extremely large values, which are in the order of magnitude $e B \sim 10$ (30) $\mathrm{GeV}^{2}$ at $\sqrt{s_{p p}}=27$ (100) TeV depending on the type of beam and centrality. The estimations for $e B$ derived within the hard-sphere approach for $\mathrm{Au}+\mathrm{Au}$ collisions at $\sqrt{s_{N N}}=0.2 \mathrm{TeV}$ [7] reasonably agree with the results from the Ultra relativistic Quantum Molecular Dynamics (UrQMD) [21] and Heavy-Ion Jet INteraction Generator (HIJING) [22] models, whereas the amplitude value of $B$ depends more weakly on centrality, and $e B(\tau)$ dependence decreases faster with $\tau$ in last cases than that for hard-sphere approach. Furthermore, there is a similar situation between the hard-sphere results and calculations from the Hadron String Dynamics (HSD) model [23] in which some smaller value of amplitude of $B$ and a faster decrease of magnetic field strength with increase 
of $\tau$ is predicted with respect to the corresponding results obtained with help of (4). The reasonable agreement between various approaches proves that the hard-sphere model can be considered to be the appropriate approach for the estimation of the time evolution of the (electro)magnetic field strength in particle collisions. Therefore, considering much higher energies studied here allows the qualitative expectation that Equation (4) provides reasonable estimations for $e B(\tau)$ in HE-LHC and FCC-hh energy domains. The previous analysis [7] also shows that $\left.(e B)\right|_{\tau_{1}} \simeq(e B)_{\max }$ at least for $\sqrt{s_{N N}}=0.1 \mathrm{TeV}$. Moreover, numerical calculations within various approaches predicting the peak in the dependence $e B(\tau)$ show that the width of the peak decreases with growth $\sqrt{s_{N N}}$. Those can expect that the values $\left.(e B)\right|_{\tau_{1}}$ obtained here for ultra-high energies (Figures 2 and 3 ) are reasonable estimations for amplitude values of the strength of magnetic field in nuclear collisions. The weakest dependence both on the beam type and on $\sqrt{s_{N N}}$ is observed for peripheral collisions, in which a dominant contribution to $\vec{B}$ comes from spectator nucleons. At fixed $\tau$ (i) the magnetic-field strength is larger for collisions of heavier nuclei; (ii) the magnetic field becomes weaker as $\sqrt{s_{N N}}$ grows within range of time $R / \sinh \left(y_{0}\right) \lesssim \tau \lesssim R$ accessible for the analytic Equation (4). The last effect is due to a faster divergence of spectator nucleons at the increase of $\sqrt{s_{N N}}$, whose contribution depends strongly on the rapidity of beam particles. These relationships between curves shown in Figures 2 and 3 for various beam types and $\sqrt{s_{N N}}$ coincide with results of previous works $[6,7]$.

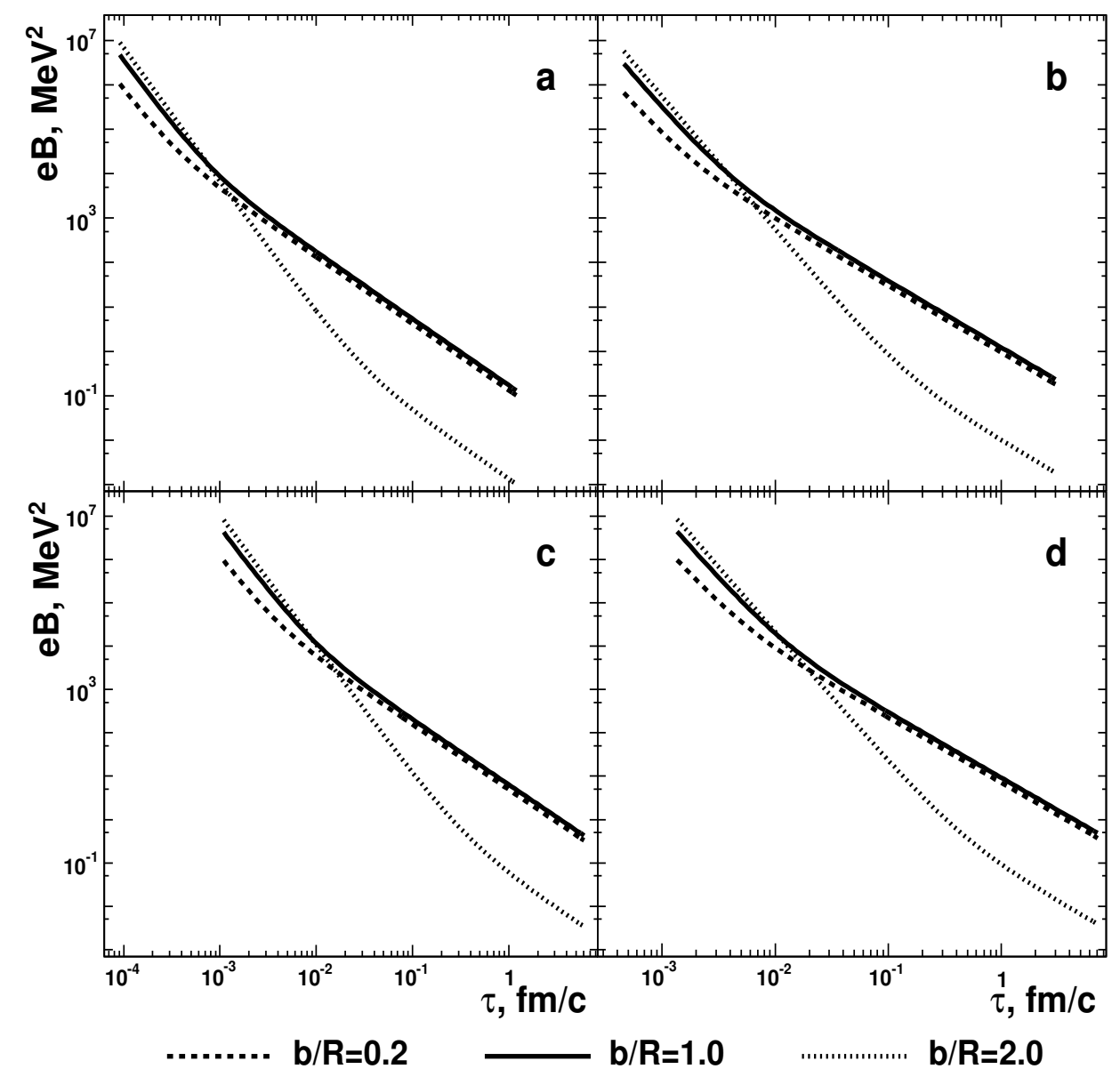

Figure 2. Dependence $e B(\tau)$ calculated with help (4) in the range $R / \sinh \left(y_{0}\right) \lesssim \tau \lesssim R$ for $p+p(\mathbf{a})$, $\mathrm{O}+\mathrm{O}(\mathbf{b}), \mathrm{Xe}+\mathrm{Xe}(\mathbf{c})$ and $\mathrm{Pb}+\mathrm{Pb}(\mathbf{d})$ collisions at nominal value $\sqrt{s_{p p}}=27 \mathrm{TeV}$ for the HE-LHC project. Dashed curves correspond to the central collisions, solid ones - semi-central, and dotted curves are for peripheral interactions. 
Here, the proton radius is estimated in accordance with the general approach $R \propto A^{1 / 3}$ for all elements from the periodic table. On the other hand, such a method provides significant overestimation of the $R_{p}$ with respect to the "preferable" Committee on Data for Science and Technology (CODATA) value $R_{c h, p}^{e p \text { CODATA value }}=0.875 \pm 0.006 \mathrm{fm}$ for charge radius of the proton [24]. Consequently, the overestimation leads to the decrease of the first term in (4) and the increase of $\tau_{1}$. This additional uncertainty is understandable for protons and a corresponding study is in progress. As observed at smaller collision energies, a consistent transition from the simplest approximation of point-like sources to the hard-sphere model and the two-component Fermi model $[25,26]$ for the nucleon-density distribution in a nucleus leads to a decrease in $B_{\max }$, especially for the first two models [27]. It seems the agreement between the model with point-like charges and the hard-sphere model is better at ultra-high energies of the HE-LHC and FCC-hh projects. Those can expect some decrease in the $e B(\tau)$ values for a more accurate Fermi model with respect to those presented here, but possibly this change will not be dramatic. The calculations are in progress for the magnetic field in ultra-high-energy particle collisions with Fermi model for nucleon distribution nucleus.

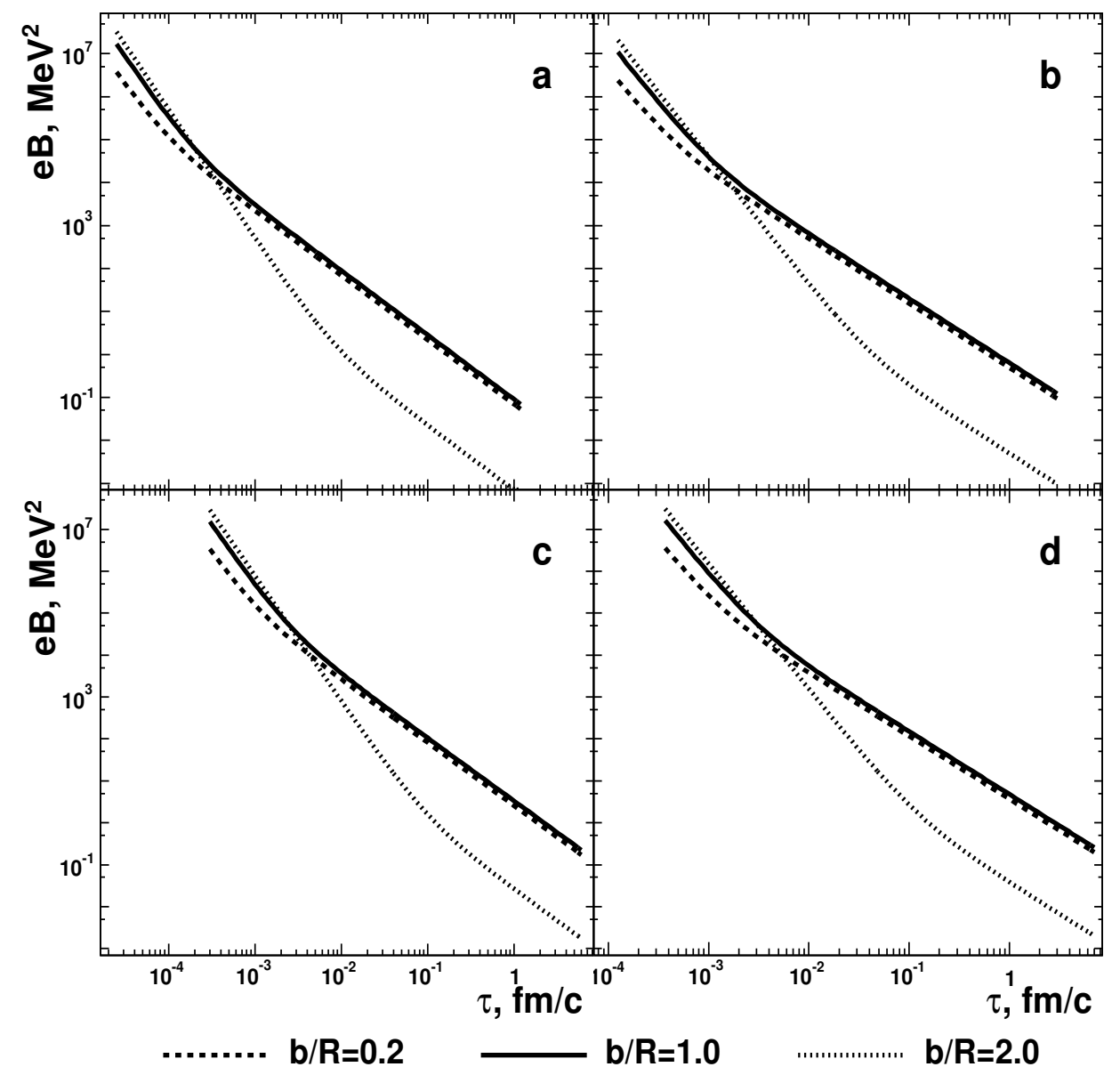

Figure 3. Dependence $e B(\tau)$ calculated with help (4) in the range $R / \sinh \left(y_{0}\right) \lesssim \tau \lesssim R$ for $p+p$ (a), $\mathrm{O}+\mathrm{O}(\mathbf{b}), \mathrm{Xe}+\mathrm{Xe}(\mathbf{c})$ and $\mathrm{Pb}+\mathrm{Pb}(\mathbf{d})$ collisions at nominal value $\sqrt{s_{p p}}=100 \mathrm{TeV}$ for the FCC-hh project. Notations used for the smooth curves are the same as in Figure 2.

Also, the time dependence of $B$ is studied for $q+q$ collisions within the approach of point-like charges. Based on the (1) the following estimations are obtained for values of the magnetic-field amplitude and characteristic time: $e B_{0} \sim 0.6(2.3) \times 10^{2} \mathrm{GeV}^{2}$ and $t_{0} \sim 1.4(0.4) \times 10^{-5} \mathrm{fm} / \mathrm{c}$ for the 
$\mathrm{Z}_{q}=1 / 3$ corresponds to the $d$-quark and $\sqrt{s_{p p}}=27(100) \mathrm{TeV}$. These estimations correspond to the relative impact parameter $x=1$.

\section{Discussion}

As discussed in [27], collisions of relativistic nuclei also generate very strong $\vec{E}$. These electromagnetic fields may have a substantial effect on multiparticle-production processes in quantum chromodynamics (QCD). The hydrodynamic properties of strongly coupled quark-gluon plasma (sQGP), together with the chiral QCD anomaly and an extremely strong external magnetic field, lead to the emergence of anomalous hydrodynamic phenomena, which are manifestations of the non-Abelian quantum nature of QCD [28]. Allied phenomena include currents flowing along the direction of the magnetic field or inner vorticity. Experimental signatures of such macroscopic manifestations of the chiral QCD anomaly are observed in nucleus-nucleus collisions as the separation of electric charges, etc.

One can note that $e B$ reaches the value on about $10^{2} \mathrm{MeV}$ in central and semi-central $p+p$ collisions for $\tau \sim 0.1 \mathrm{fm} / c$ at energy of HE-LHC (Figure 2a) and FCC-hh (Figure 3a). This value corresponds in order of magnitude to the range of low boundary for the strength of magnetic field at which experimental manifestation of chiral magnetic effect (CME) appears $(e B)_{\min }^{\mathrm{CME}} \sim\left(\alpha_{S} T\right)^{2} \sim$ $10^{2}-10^{3} \mathrm{MeV}^{2}$ [3]. On the other hand, the $\tau \sim 0.1 \mathrm{fm} / c$ agrees with the estimations for the onset the thermalization of the plasma into a sQGP. Moreover, the magnetic-field lifetime increases sharply upon taking into account the conductivity of matter and its expansion [17]. Therefore, the present investigation of the magnetic field within the hard-sphere model indicates that the HE-LHC and FCC-hh projects can provide the novel possibility of studying the chiral effects, e.g., CME in $p+p$ collisions. One can expect that the background effects in $p+p$ events will be significantly weaker than that in nucleus-nucleus collisions at the same energy. Also, extremely large values of $B$ at ultra-high energies and high luminosities of HE-LHC and FCC-hh can provide the opportunity to study flavor dependence of the $\mathcal{P} / \mathcal{C P}$ violation with the help of the azimuthal correlations of various particle species. Thus, experimental study of topology of $Q C D$ vacuum can be one of the focuses for studies of bulk properties at the HE-LHC and FCC. Furthermore, the $e B \gtrsim 10^{-2} \mathrm{GeV}^{2}$ would also have a profound effect on the breaking of the $S U(2) \times S U(2)$ chiral symmetry of the strong interactions [29]. As seen in Figures 2 and 3, the strength of the magnetic field achieves such values within the hard-sphere model at $\tau \lesssim 10^{-3} \mathrm{fm} / c$ in $p+p$ (Figures $2 \mathrm{a}$ and $3 \mathrm{a}$ ) and $\mathrm{O}+\mathrm{O}$ (Figures $2 \mathrm{~b}$ and $3 \mathrm{~b}$ ) interactions and at significantly larger times $\tau \lesssim 10^{-2}$ in collisions of heavier ions $\mathrm{Xe}+\mathrm{Xe}$ (Figures $2 \mathrm{c}$ and $3 \mathrm{c}$ ), $\mathrm{Pb}+\mathrm{Pb}$ (Figures $2 \mathrm{~d}$ and $3 \mathrm{~d}$ ). Thus, the extremely strong magnetic field can affect chirality during the non-equilibrium very early stages of space-time evolution of the final-state strongly interacting matter.

The HE-LHC, FCC-hh facilities open the novel opportunity to study polarization phenomena in hot environments, in particular the precise measurements of the difference in polarization of primary $\Lambda$ and $\bar{\Lambda}$ and polarization of heavier hyperons (for instance, $\Sigma$ ). Considered extremely strong, $e B \sim 10$ $\mathrm{GeV}^{2}$ will provide important changes for the behavior of quarkonium and, perhaps, heavier particles and states in particular $\bar{t} t$. Also, electromagnetic fields created in particle collisions at ultra-relativistic energies can be useful for the study of fundamental properties of theory, namely non-linear or non-commutative features of quantum electrodynamics (QED), e.g., with help of the light-by-light scattering, production of the magnetic monopoles by the electromagnetic dual of Schwinger pair creation, etc. [30]. However, these semi-qualitative suggestions should be verified by additional quantitative and detailed analysis.

In [31] the possible effect of Bose-Einstein condensation in $p+p$ and nucleus-nucleus collisions at FCC-hh energies were considered in detail. The closely related topic is the study of influence of the very short pulse of the extremely strong Abelian (electro)magnetic field on particle production, in particular, pion condensation in external field [32]. Furthermore, as shown above, the amplitude value of the magnetic field expected for $q+q$ collisions is $e B_{0} \sim 1.0(4.0) \times 10^{22} \mathrm{G}$ for the $\mathrm{Z}_{q}=1 / 3$ 
corresponding to the $d$-quark and $\sqrt{s_{p p}}=27$ (100) TeV. These values are close in order to provide magnitude to the estimation for strength of $\vec{B}$ at which $W$ boson condensation occurs [33]. The amplitude values of $e B$ for any considered interactions ( $A+A$ from Table 1 and $q+q$ collisions) are in the range of so-called very intense magnetic fields $\left(10^{18}-10^{24} \mathrm{G}\right)$ which can be generated in the early universe [29]. Such $B$ can influence the structure of the electroweak vacuum and on the properties of corresponding phase transition. Therefore, the extremely strong $B$ at HE-LHC and FCC-hh energies can influence the electroweak processes.

\section{Conclusions}

Summarizing the study, one can draw the following conclusions.

Collisions of relativistic particles are a source of the strongest electromagnetic field known in nature. For the first time, the estimations for absolute value of magnetic field are obtained within various approaches for proton and nuclear beams at ultra-high energies corresponding to the HE-LHC and FCC-hh projects. The analytic approaches used for estimation of strength of the magnetic field do not consider the possible influence of the matter created in final state, i.e., the approaches correspond to the vacuum conditions. The model with point-like charges predicts the peak value of $e B$ about $(13-20) \mathrm{GeV}^{2}$ at $\sqrt{s_{p p}}=27 \mathrm{TeV}$ and $(49-71)$ at $\sqrt{s_{p p}}=100 \mathrm{TeV}$ while the more realistic hard-sphere approach provides $e B \sim 10$ and $30 \mathrm{GeV}^{2}$. The strength of magnetic field rapidly decreases with time and increases with growth of atomic number. The amplitude for $e B$ is estimated at level $60(200) \mathrm{GeV}^{2}$ in quark-quark collisions for charges corresponding to $d$-quark at nominal $\sqrt{s_{p p}}=27$ (100) TeV.

The extremely strong (electro)magnetic field expected at HE-LHC and FCC-hh can influence strong and electroweak interaction processes. In particular, the principle possibilities appear for the study of chiral magnetic effect in proton-proton collisions, for $W$ boson condensation and for manifestation of non-commutative features of the quantum electrodynamics. Further development of theoretical and experimental methods is of crucial importance for drawing more definitive conclusions for these qualitative suggestions.

Funding: This work was supported partly by NRNU MEPhI Academic Excellence Project (contract No 02.a03.21.0005, 27.08.2013).

Conflicts of Interest: The author declares no conflict of interest.

\section{Abbreviations}

The following abbreviations are used in this manuscript:

CODATA Committee on Data for Science and Technology

FCC Integrated project of the Future Circular Collider

FCC-hh Work mode of the FCC with proton and nuclear beams

HE-LHC Project of the future high-energy hadron collider based on the present Large Hadron Collider

HIJING Heavy-Ion Jet INteraction Generator

HSD Hadron String Dynamics

QCD Quantum chromodynamics

QED Quantum electrodynamics

UrQMD Ultra relativistic Quantum Molecular Dynamics

\section{References}

1. Minakata, H.; Müller, B. Disoriented chiral condensate and strong electromagnetic field. Phys. Lett. B 1996, 377, 135-139. [CrossRef]

2. Kharzeev, D.E.; Warringa, H.J. Chiral magnetic conductivity. Phys. Rev. D 2009, 80, 034028. [CrossRef]

3. Kharzeev, D.E.; McLerran, L.D.; Warringa, H.J. The effects of topological charge change in heavy ion collisions: "Event by event $\mathcal{P}$ and $\mathcal{C P}$ violation". Nucl. Phys. A 2008, 803, 227-253. [CrossRef]

4. Kharzeev, D. Can gluons trace baryon number? Phys. Lett. B 1996, 378, 238-246. [CrossRef] 
5. Tanabashi, M.; Hagiwara, K.; Hikasa, K.; Nakamura, K.; Sumino, Y.; Takahashi, F.; Tanaka, J.; Agashe, K.; Aielli, G.; Amsler, C.; et al. Review of particle physics. Phys. Rev. D 2018, 98, 030001. [CrossRef]

6. Okorokov, V.A. Estimation of $\mathcal{P}$-odd correlators in heavy ion collisions at RHIC energies $62.4-200 \mathrm{GeV}$. arXiv 2009, arXiv:0908.2522.

7. Okorokov, V.A. Dependence of the charge asymmetry parameters on the initial energy in heavy ion collisions. Yad. Fiz. Inzhin. 2013, 4, 805.

8. Abada, A.; Abbrescia, M.; AbdusSalam, S.S.; Abdyukhanov, I.; Abelleira Fernandez, J.; Abramov, A.; Aburaia, M.; Acar, A.O.; Adzic, P.R.; Agrawal, P.; et al. Future Circular Collider Study. Volume 4: The High Energy LHC (HE-LHC) Conceptual Design Report; Zimmermann, F., Benedikt, M., Capeans, M., Cerutti, F., Goddard, B., Gutleber, J., Jiménez, J.M., Mangano, M., Mertens, V., Osborne, J., et al., Eds.; CERN Accelerator Reports CERN-ACC-2018-0059; CERN: Geneva, Switzerland, 2018.

9. Abada, A.; Abbrescia, M.; AbdusSalam, S.S.; Abdyukhanov, I.; Abelleira Fernandez, J.; Abramov, A.; Aburaia, M.; Acar, A.O.; Adzic, P.R.; Agrawal, P.; et al. Future Circular Collider Study. Volume 3: The Hadron Collider (FCC-hh) Conceptual Design Report; Benedikt, M., Capeans, M., Cerutti, F., Goddard, B., Gutleber, J., Jiménez, J.M., Mangano, M., Mertens, V., Osborne, J., Otto, T., et al., Eds.; CERN Accelerator Reports CERN-ACC-2018-0058; CERN: Geneva, Switzerland, 2018.

10. Aichelin, J.; Angerami, A.; Apolinario, L.; Arleo, F.; Armesto, N.; Arnaldi, R.; Arslandok, M.; Azzi, P.; Bailhache, R.; Bass, S.A.; et al. Future Physics Opportunities for High-Density QCD at the LHC with Heavy-Ion and Proton Beams. Report from Working Group 5 on the Physics of the HL-LHC, and Perspectivies at the HE-LHC; Citron, Z., Dainese, A., Grosse-Oetringhaus, J.F., Jowett, J.M., Lee, Y.-J., Wiedemann, U.A., Winn, M., Eds.; CERN Report CERN-LPCC-2018-07; CERN: Geneva, Switzerland, 2018.

11. Valentin, L. Subatomic Physics: Nuclei and Particles; Hermann: Paris, France, 1982.

12. Mukhin, K.N. Experimental Nuclear Physics; Mir: Moscow, Russia, 1987.

13. Sarkisyan, E.K.G.; Sakharov, A.S. On Similarities of Bulk Observables in Nuclear and Particle Collisions; CERN Report CERN-PH-TH-2004-213; arXiv:hep-ph/0410324; CERN: Geneva, Switzerland, 2004.

14. Sarkisyan, E.K.G.; Sakharov, A.S. Multihadron production features in different reactions. AIP Conf. Proc. 2006, 828, 35-41.

15. Sarkisyan, E.K.G.; Sakharov, A.S. Relating multihadron production in hadronic and nuclear collisions. Eur. Phys. J. C 2010, 70, 533-541. [CrossRef]

16. Sarkisyan, E.K.G.; Mishra, A.N.; Sahoo, R.; Sakharov, A.S. Multihadron production dynamics exploring the energy balance in hadronic and nuclear collisions. Phys. Rev. D 2016, 93, 054046. [CrossRef]

17. Tuchin, K. Particle production in strong electromagnetic fields in relativistic heavy-ion collisions. Adv. High Energy Phys. 2013, 2013, 490495. [CrossRef]

18. Huang, X.G. Electromagnetic fields and anomalous transports in heavy-ion collisions-A pedagogical review. Rep. Prog. Phys. 2016, 79, 076302. [CrossRef] [PubMed]

19. Paquet, J.-F. Overview of electromagnetic probe production in ultra-relativistic heavy ion collisions. J. Phys. Conf. Ser. 2017, 832, 012035. [CrossRef]

20. Zhong, Y.; Yang, C.-B.; Cai, X; Feng, S.-Q. A systematic study of magnetic field in relativistic heavy-ion collisions in the RHIC and LHC energy regions. Adv. High Energy Phys. 2014, 2014, 193039. [CrossRef]

21. Skokov, V.V.; Illarionov, A.Yu.; Toneev V.D. Estimate of the magnetic field strength in heavy-ion collisions. Int. J. Mod. Phys. A 2009, 24, 5925-5932. [CrossRef]

22. Deng, W.-T.; Huang X.-G. Event-by-event generation of electromagnetic fields in heavyion collisions. Phys. Rev. D 2012, 85, 044907.

23. Voronyuk, V.; Toneev, V.D.; Cassing, W.; Bratkovskaya, E.L.; Konchakovski, V.P.; Voloshin, S.A. Electromagnetic field evolution in relativistic heavy-ion collisions. Phys. Rev. C 2011, 83, 054911. [CrossRef]

24. Petrov, V.A.; Okorokov, V.A. The size seems to matter or where lies the "asymptopia"? Int. J. Mod. Phys. A 2018, 33, 1850077. [CrossRef]

25. Okorokov, V.; Parfenov, P. Particle correlators and possible local parity violation in nuclear collisions. J. Phys. Conf. Ser. 2016, 668, 012129. [CrossRef]

26. Okorokov, V.; Parfenov, P. Charge-dependent azimuthal correlations of secondary particles in heavy ion collisions. J. Phys. Conf. Ser. 2016, 675, 022021. [CrossRef]

27. Okorokov, V.A. Chiral effects in nucleus-nucleus collisions: Experimental review. Phys. At. Nucl. 2017, 80, 1133-1140. [CrossRef] 
28. Kharzeev, D.E.; Liao, J.; Voloshin, S.A.; Wang, G. Chiral magnetic and vortical effects in high-energy nuclear collisions-A status report. Prog. Part. Nucl. Phys. 2016, 88, 1-28. [CrossRef]

29. Grasso, D.; Rubinstein, H.R. Magnetic fields in the early Universe. Phys. Rep. 2001, 348, 163-266. [CrossRef]

30. Bruce, R.; d'Enterria, D.; de Roeck, A.; Drewes, M.; Farrar, G.R.; Giammanco, A.; Gould, O.; Hajer, J.; Harland-Lang, L.; Heisig, J.; et al. New physics searches with heavy-ion collisions at the LHC. arXiv 2018, arXiv:1812.07688.

31. Okorokov, V.A. Geometry and space-time extent of pion emission region at FCC energies. Adv. High Energy Phys. 2016, 2016, 5972709. [CrossRef]

32. Liu, Y.; Zahed, I. Pion condensation by rotation in a vagnetic field. Phys. Rev. Lett. 2018, 120, 032001. [CrossRef] [PubMed]

33. Ambjorn, J.; Olesen, P. W-condensate formation in high energy collisions. Phys. Lett. B 1991, 257, $201-206$. [CrossRef]

(C) 2019 by the author. Licensee MDPI, Basel, Switzerland. This article is an open access article distributed under the terms and conditions of the Creative Commons Attribution (CC BY) license (http:/ / creativecommons.org/licenses/by/4.0/). 